\title{
Investigating State Reconstruction from Scarce Synchronized Phasor Measurements
}

\author{
Mevludin Glavic, Senior Member, IEEE
}

\author{
Thierry Van Cutsem, Fellow, IEEE
}

\begin{abstract}
Synchronized phasor measurements can potentially track the system dynamics between two classical state estimations. However, in the PMU configurations available nowadays and in the near future, those measurements are too scarce for the whole system state to be estimated. Therefore, we investigate the possibility to reconstruct coherent, time-synchronized system states from the available PMU data. State reconstruction is formulated as an optimization problem. The objective is to minimize, in the space of bus powers, the distance between the reconstructed state and the last state estimate provided by a standard state estimator. PMU data are imposed as equality constraints. Furthermore, the placement of PMUs near generators is advocated for higher accuracy of state reconstruction. The performance and potential benefits of the approach are illustrated by processing snapshots obtained from detailed time simulation of a test system subject to a disturbance and corrective actions.
\end{abstract}

Index Terms-state reconstruction, synchronized phasor measurements, constrained optimization, unobservability, situational awareness, state estimation

\section{INTRODUCTION}

\section{A. Background and motivation}

The Phasor Measurement Unit (PMU) is an accurately time synchronized power system instrument able to gather voltage and current phasors at high rate (30-60 samples/second) [1], [2]. Supported by advances in computational facilities, networking infrastructure and communications, this technology have opened new perspectives for designing wide-area monitoring, protection and control systems. Present and potential applications of synchronized phasor measurements range from mere monitoring to tracking system dynamics in real-time [1], [2].

It is likely that in some future, all measurement devices will be provided with high precision time tags making the whole system observable by these devices [1], [2], [3], [4], [5]. These new technological solutions are available in presentday power systems but with scarce PMUs since the upgrade of existing power system infrastructures requires investments in these technologies and only incremental upgrades are realistic. Consequently, present-day power systems are still far from having a rich synchronized measurement configuration.

A natural application of PMUs, even more if scarce, is to enhance traditional state estimators based on SCADA (Supervisory Control And Data Acquisition) measurements [1], [2].

M. Glavic (glavic@montefiore.ulg.ac.be) is visiting professor at the Dept. of Electrical Engineering and Computer Science (Montefiore Institute) of the University of Liège, Belgium.

T. Van Cutsem (t.vancutsem@ulg.ac.be) is a research director of the Fund for Scientific Research (FNRS) and adjunct professor at the Dept. of Electrical Engineering and Computer Science (Montefiore Institute) of the University of Liège, Belgium.
Traditional state estimators run every few minutes and provide an average picture of the system state due to time skew in measurement set. Synchronized measurement snapshots taken in almost the same time window as the classical measurements can be used to reinforce the redundancy of the latter. From a practical viewpoint, it may be advantageous to post-process the PMU measurements separately so that the state estimation software is not modified [6].

On the other hand, synchronized phasor measurements can potentially track the system dynamics between two classical state estimations. However, scarce PMUs do not allow determining the whole system state. Consequently, one of the challenges in effective exploitation of existing or near-future PMU configurations is to somewhat "reconstruct" coherent time-synchronized system states from the available PMU data. This challenge has been recognized by power system practitioners and research community and efforts have been undertaken to use PMU data to increase situational awareness [3], [4], [7], [8], [9].

In this paper, we formulate state reconstruction as an optimization problem with linear equality constraints. The objective is to minimize, in the space of bus powers, the distance between the reconstructed state and the last state estimate provided by a standard state estimator. The constraints relate to zero current injections and available phasor measurements. Furthermore, we advocate the placement of PMUs at generator buses for increased accuracy of the state reconstruction.

The paper is structured as follows. Some related works are discussed in Section II. The principle of state reconstruction and mathematical formulation of the problem are given in Section III. PMU placement for improved accuracy of state reconstruction is discussed in Section IV. Section V presents the results of the approach tested on the Nordic32 test system. By way of conclusion, Section VI summarizes the approach and the main results, and outlines potential benefits and ongoing investigations.

\section{RELATED WORKS}

This section reviews previous and current works dealing more or less closely with state estimation from scarce data, classified in three categories.

\section{A. Increased situational awareness [3], [4], [7], [8], [9]}

A hybrid power flow model that combines PMU measurement data and power flow equations to increase situational awareness at the system level, was introduced in [7]. Essentially, the approach complements observable islands 
with assumed power flow conditions. An observable island is defined as a set of buses whose states can be directly computed from PMU measurements [6], [7]. The power flow model includes multiple swing buses (all observable buses whose voltage phasors are known), $P Q V \theta$ buses (all observable buses where voltage and current phasors are known), $P Q$ and $P V$ buses as in a traditional power flow model, and the socalled nought buses (connected directly to $P Q V \theta$ buses and where all variables are unknown). Solving this hybrid power flow problem yields a system state that matches the available synchronized phasor measurements [7].

A PMU morphed power flow approach was introduced in [8], [9]. The approach starts from a solved power flow and proceeds by matching this known solution to the small number of PMU measurements. This involves solving a linear programming problem with known system values (some generator outputs, total load in some areas, etc.) treated as constants and unknown values (the other generator outputs, the other area loads, etc.) treated as controls [8], [9]. The set of binding constraints is set up to include the PMU bus angle measurements as equality constraints while the objective is to minimize control costs.

In order to deal with situations where only a limited number of PMUs are available, Refs. [3], [4] introduced the concept of interpolation of states of unobservable buses: using the bus admittance matrix, a matrix of interpolation coefficients is derived relating the unobservable voltages to the observable ones. This concept, together with the interpolation updating based on SCADA measurements, is used for hybrid state estimation. Injections at unobservable buses are treated as equivalent load admittances. It is suggested to update the interpolation coefficients as soon as a new system snapshot is available from conventional state estimation. The voltages at unobservable buses are coherent with the set of phasor measurements.

\section{B. Harmonic state estimation [10]}

Power system harmonic state estimation aims at locating major harmonic sources and estimating the distribution of harmonic voltages and currents. This requires to cope with a lack of measurements. The approach in [10] uses a limited number of phasor measurements (fewer than unknown state variables) to this purpose. The problem is formulated as a constrained sparsity-maximization problem based on $L_{1}$-norm minimization to deal with the underdetermined set of linear equations, and is solved efficiently by an equivalent linear programming.

\section{Other related works [11], [12], [13]}

Reference [11] also deals with the problem of determining an approximate state from a limited number of measurements. Although PMUs are not specifically considered, the idea can be extended to those measurements. The problem of approximate state computation is set as a nonlinear constrained optimization problem. The objective function reflects deviations from desired operating conditions (i.e. an ideal operating point with voltage magnitudes near $1 \mathrm{pu}$ and small phase angle differences across lines) and minimizes active and reactive losses. Standard power flow equations are set as equality constraints while inequality constraints involve load active powers (ranked according to size), active and reactive power generations and bus voltage magnitudes and phase angles.

Although not involving PMU data, state estimation in distribution systems typically also faces the problem of scarce real-time measurements. Most of the time this is taken care of by adding pseudo-measurements based on load forecasts. In Ref. [12] the impact of erroneous forecasts is mitigated by adjusting the loads to match the feeder measurements.

A similar problem is met by traditional state estimation of transmission systems in the presence of unobservable areas. An approach consists in introducing pseudo-measurements. In order to improve the estimation of unobservable parts, the use of optimal power flow was proposed in [13]. The objective is to satisfy in the least-square sense the pseudo-measurements in unobservable areas. The constraints include: power balance, power injections, state variables. Line flow constraints proved to improve the overall state estimation accuracy.

These works are related to the approach presented in this paper in so far as they either adjust loads to match the available measurements [12] or resort to optimization to better estimate unobservable areas [13].

\section{PRINCIPLE AND MATHEMATICAL FORMULATION OF STATE RECONSTRUCTION}

Let the network be modelled by the voltage-current relationships stemming from the bus admittance matrix formulation. Decomposing the vectors of bus voltages and nodal currents into their real and imaginary parts, the network equations take on the matrix form:

$$
\begin{aligned}
& G v_{\mathbf{x}}-\mathbf{B} \mathbf{v}_{\mathbf{y}}-\mathbf{i}_{\mathbf{x}}=\mathbf{0} \\
& \mathbf{B} \mathbf{v}_{\mathbf{x}}+\mathbf{G v}_{\mathbf{y}}-\mathbf{i}_{\mathbf{y}}=\mathbf{0}
\end{aligned}
$$

where $\mathbf{v}_{\mathbf{x}}, \mathbf{v}_{\mathbf{y}}, \mathbf{i}_{\mathbf{x}}$ and $\mathbf{i}_{\mathbf{y}}$ are vectors of rectangular components of voltages and currents, while $\mathbf{G}$ and $\mathbf{B}$ are the real and imaginary parts of the bus admittance matrix, respectively.

Our objective is to compute a coherent, time-synchronized, system state that satisfies a number of PMU data. The latter involve some components of $\mathbf{v}_{\mathbf{x}}, \mathbf{v}_{\mathbf{y}}, \mathbf{i}_{\mathbf{x}}, \mathbf{i}_{\mathbf{y}}$ according to:

$$
\mathbf{A}\left[\begin{array}{c}
\mathbf{v}_{\mathbf{x}} \\
\mathbf{v}_{\mathbf{y}} \\
\mathbf{i}_{\mathbf{x}} \\
\mathbf{i}_{\mathbf{y}}
\end{array}\right]=\mathbf{z}
$$

where $\mathbf{z}$ is the vector of measured values. The corresponding rows of $\mathbf{A}$ are unit vectors with the nonzero (unit) entry corresponding to the measured voltage or current component. We also include in (2) the zero current injection information at "transit" buses with neither load nor generation connected. The corresponding component of $\mathbf{z}$ is zero.

We assume that all measurements in $\mathbf{z}$ are critical, i.e. removing any of them would decrease the number of bus voltages that can be computed from the PMU and zero injection data. Adaptations to handle redundant measurements are straightforward. 
Note that each voltage or current phasor measurement, and each zero current injection yields two equations in (2) corresponding to respectively its real and imaginary parts.

We assume that the PMU configuration provides scarce measurements, which do not make the system observable, i.e. there are less equations $(1,2)$ than voltages and currents to be estimated. There is thus an infinite number of states satisfying the available set of synchronized measurements. The standard way of solving this indeterminacy, used in many engineering disciplines [14], consists of determining the state vector with minimum $\left(L_{0}, L_{1}\right.$ or $\left.L_{2}\right)$ norm.

We adopt a similar reasoning and remove the indeterminacy by considering the state closest to the estimate provided by the last execution of a standard state estimator. Furthermore, for reasons discussed in the next section, we minimize the distance to that reference in the space of bus (active and reactive) powers. This leads to minimizing:

$$
\sum_{i \in \mathcal{N}}\left(P_{i}^{0}-P_{i}\right)^{2}+\sum_{i \in \mathcal{N}}\left(Q_{i}^{0}-Q_{i}\right)^{2}
$$

where $P_{i}^{0}$ (resp. $Q_{i}^{0}$ ) is the active (resp. reactive) power at the $i$-th bus in the reference state, $P_{i}$ (resp. $Q_{i}$ ) the corresponding reconstructed value, and $\mathcal{N}$ is the set of involved buses. The choice of the latter is also discussed in the next section. At most, it might include all buses except those with zero injection, which are already involved in (2). Although alternatives may be envisaged, our preference goes to the Euclidian distance (3) which has the advantage of distributing the discrepancies between reference and reconstructed states more evenly over the system.

Alternatively, the minimization of (3) can be seen as a leastsquare estimation of the unknown $P_{i}, Q_{i}$ powers, with the reference load powers $P_{i}^{o}, Q_{i}^{o}$ used as pseudo-measurements.

Expressing $P_{i}$ and $Q_{i}$ in terms of voltage and current components in (3) leads to the following constrained optimization problem:

$$
\begin{aligned}
\min _{\mathbf{v}_{\mathbf{x}}, \mathbf{v}_{\mathbf{y}}, \mathbf{i}_{\mathbf{x}}, \mathbf{i}_{\mathbf{y}}} & \sum_{i \in \mathcal{N}}\left(P_{i}^{0}-v_{x i} i_{x i}-v_{y i} i_{y i}\right)^{2}+ \\
& +\sum_{i \in \mathcal{N}}\left(Q_{i}^{0}-v_{y i} i_{x i}+v_{x i} i_{y i}\right)^{2} \\
\text { subject to : } & \mathbf{G}_{\mathbf{x}}-\mathbf{B v}_{\mathbf{y}}-\mathbf{i}_{\mathbf{x}}=\mathbf{0} \\
& \mathbf{B} \mathbf{v}_{\mathbf{x}}+\mathbf{G v}_{\mathbf{y}}-\mathbf{i}_{\mathbf{y}}=\mathbf{0} \\
& \mathbf{A}\left[\begin{array}{c}
\mathbf{v}_{\mathbf{x}} \\
\mathbf{v}_{\mathbf{y}} \\
\mathbf{i}_{\mathbf{x}} \\
\mathbf{i}_{\mathbf{y}}
\end{array}\right]-\mathbf{z}=\mathbf{0}
\end{aligned}
$$

The above optimization has two interesting features. Firstly, all constraints are linear, nonlinearity is present in the objective only. The linearity of (4d) stems from the use of phasor measurements. There would be no advantage in converting them to nonlinear power measurements. Secondly, the G, B and $\mathbf{A}$ matrices are very sparse. Both features facilitate the solving of that optimization problem.

Additional information on the system state could be incorporated in the form of inequality constraints; however, the associated benefits should be balanced against the increased computational complexity.

\section{ON THE PLACEMENT OF PMUS}

\section{A. Motivation for monitoring generators}

Different PMU configurations are expected to yield different accuracies for the solution of (4). So far, our investigations have shown that monitoring generators through PMUs yields a significantly more accurate state. This may be explained as follows.

A situation of interest for exploiting synchronized phasor measurements is when a disturbance takes place after the execution of the classical state estimator. In such a case, load powers change owing to their sensitivity to voltage and frequency. However, this change is usually in the order of a few percents. On the contrary, the powers produced by generators may vary significantly under the effect a disturbance, depending on the performance of primary voltage and frequency control. For instance, the outage of transmission or generation equipments is reflected in the reactive powers of voltage controlled generators. Thus, it makes sense to collect measurements of those changing values.

In order to synchronously monitor the outputs of the various generators, two PMU configurations can be thought of:

1) placed at the generator buses: each PMU provides the generator voltage and the current in the step-up transformer;

2) placed at the high-voltage ends of the step-up transformers: each PMU provides the bus voltage, the current in the step-up transformer as well as the current in each line connected to the bus of concern ${ }^{1}$.

Configuration 2 is more common in practice. It is also richer since it makes observable a larger number of bus voltages [1], [15]. Nevertheless, configuration 1 was also considered in this work in order to make the tests more stringent.

Whatever the configuration, for each generator provided with a PMU, the corresponding measurements are included in (4d) while the active and reactive power terms relative to that generator are removed from (4a). Thus, if all generators are equipped with PMUs, the set $\mathcal{N}$ in (4a) includes load buses only.

\section{B. Supporting simple example}

We further illustrate the benefit of monitoring all key generators through a very simple example.

We consider the 5-bus system shown in Fig. 1, including one load, two generators, and two buses with zero current injection. The reference state is obtained as the solution of a power flow problem with the data shown in the left part of Table I. Then, a disturbed state is obtained by increasing the load by $10 \%$. The active power increase is fully covered by the generator at bus 2 , while both generators keep their terminal voltages constant. This leads to the disturbed state shown in the right part of Table I.

${ }^{1}$ provided, of course, that there are enough communication channels to transmit all the synchronized phasor data 


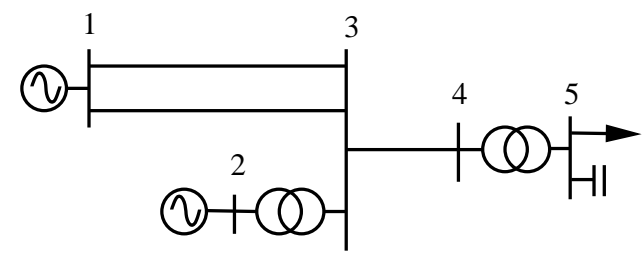

Fig. 1. Example 5-bus system

TABLE I

DATA OF REFERENCE AND DISTURBED STATES

\begin{tabular}{|c||c|c||c|c|}
\hline \multicolumn{1}{|c||}{} & \multicolumn{2}{c||}{ reference state } & \multicolumn{2}{c|}{ disturbed state } \\
& $\mathrm{P}(\mathrm{MW})$ & $\mathrm{Q}(\mathrm{Mvar})$ & $\mathrm{P}(\mathrm{MW})$ & $\mathrm{Q}(\mathrm{Mvar})$ \\
\hline generated at bus 1 & 0. & 61.27 & 0 & 66.00 \\
generated at bus 2 & 400.00 & 51.30 & 440.00 & 61.48 \\
consumed at bus 5 & 400.00 & 80.00 & 440.00 & 88.00 \\
\hline
\end{tabular}

We consider the reconstruction of the disturbed state from the bus powers of the reference state and the synchronized phasor measurements provided by respectively:

A: a single PMU located at bus 1 , and

B: 2 PMUs, located at buses 1 and 2 .

Thus, with configuration A, the objective function (3) takes on the form:

$$
\left(P_{2}^{0}-P_{2}\right)^{2}+\left(Q_{2}^{0}-Q_{2}\right)^{2}+\left(P_{5}^{0}-P_{5}\right)^{2}+\left(Q_{5}^{0}-Q_{5}\right)^{2}
$$

with the values of $P_{2}^{0}, Q_{2}^{0}, P_{5}^{0}$ and $Q_{5}^{0}$ taken from columns 2 and 3 in Table I, while with configuration $\mathrm{B}$, the objective amounts to the last two terms only.

The results are summarized in Table II in terms of reconstructed active and reactive powers for both configurations.

TABLE II

RECONSTRUCTED POWERS FOR TWO PMU CONFIGURATIONS

\begin{tabular}{|c||c|c||c|c|}
\hline \multicolumn{1}{|c||}{} & \multicolumn{2}{c||}{ configuration A } & \multicolumn{2}{c|}{ configuration B } \\
& P (MW) & Q (Mvar) & P (MW) & Q (Mvar) \\
\hline generated at bus 1 & 0. & 66.00 & 0 & 66.00 \\
generated at bus 2 & 400.18 & 49.08 & 440.00 & 61.48 \\
consumed at bus 5 & 400.18 & 82.26 & 440.00 & 87.98 \\
\hline
\end{tabular}

Comparing the second and third columns of Table II with the fourth and fifth columns of Table I shows non negligible errors in reconstructed powers of the load at bus 5 and the generation at bus 2 , when using configuration A. These errors can be explained as follows.

At bus 1, the power is forced to have a correct value to satisfy the PMU data available at that bus. This does not hold true at buses 2 and 5 where the powers are left to vary; in the absence of more information, the reconstructed values closely approach their reference values, which minimizes the objective function (5). In fact, the reconstructed load at bus 5 can approach its reference value $400 \mathrm{MW}$, which is $40 \mathrm{MW}$ below the exact value, because at the same time the generation at bus 2 can also settle $40 \mathrm{MW}$ below its exact value. And all this without affecting the production at bus 1 .

This is no longer possible when PMU data are available at bus 2. In this case, the results in the last two columns of Table II show that the reconstructed state is very close to the exact value. This confirms the relevance of providing generators with PMUs for accurate state reconstruction.

\section{Simulation Results}

\section{A. Test system and simulation procedure}

We report on results obtained with a variant of the Nordic32 test system already used in [22]. The one-line diagram of this 52-bus, 20-machine system is shown in Fig. 2.

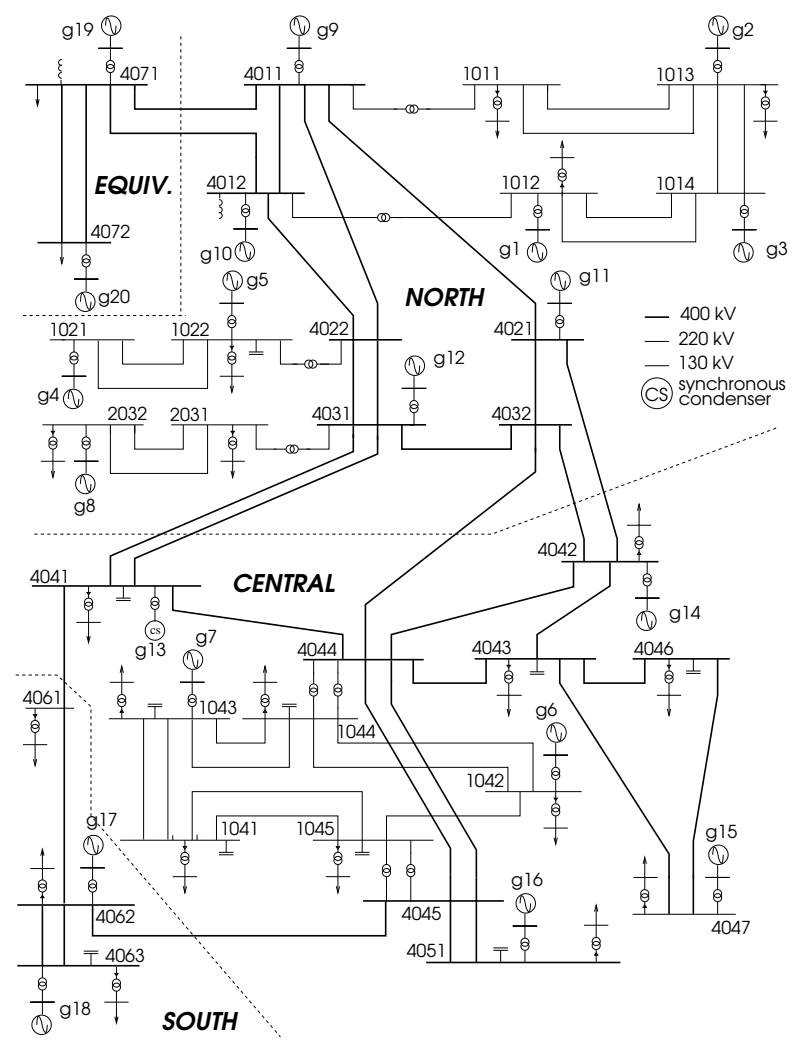

Fig. 2. Nordic32 test system

The model includes a detailed representation of each synchronous machine, and simple models of speed governors, hydro and steam turbines, automatic voltage regulators and OverExcitation Limiters (OELs). Only the hydro-plant generators in the North and Equiv areas (see Fig. 2) are equipped with speed governors, and frequency is thus controllers by those generators only. Loads behave as constant current for the active power and constant impedance for the reactive power. Each load is fed through a transformer with automatic Load Tap Changer (LTC); the various LTCs act with various delays.

Time-domain simulations of the model have been performed with the Simulink-based variable-step integration software outlined in [16].

Two scenarios have been considered:

1) Scenario A: a slow load increase in the Central part of the system (namely at buses 1041, 1042, 1043, 1044, and 1045) with a step change of the load demand at bus 1041 , taking place at $t=100 \mathrm{~s}$;

2) Scenario B: tripping of line $4032-4044$ at $t=6 \mathrm{~s}$, followed by load shedding at buses 1041, 1043, and 1045 , at respectively $t=40,50$ and $60 \mathrm{~s}$. This load 
curtailment stabilizes the otherwise long-term voltage unstable evolution [22].

In both scenarios, LTCs and OELs act in response to the disturbance.

PMUs providing the terminal voltages and the currents of generators have been assumed. As already mentioned, it would be more realistic to place them at the high-voltage side of the step-up transformers. However, this would yield too good a coverage of the buses in this small test system. Table III details the generators selected in the three configurations referred to in the remaining of the paper. We assume that we are primarily interested in the Central region, where load powers increase of Scenario A and whose voltages are the most affected by the disturbance of Scenario B. The three measurement configurations detailed in Table III were selected to progressively reduce the coverage of the parts of the system in which we are not interested. Configuration 3 is particularly challenging in this respect.

TABLE III

GENERATORS PROVIDED WITH PMUS

\begin{tabular}{|c|l|}
\hline configuration & generators \\
\hline 1 & all 20 \\
2 & g6, g7, g11, g12, g13, g14, g15, g16 \\
3 & g6, g7, g14, g15, g16 \\
\hline
\end{tabular}

The phasor data were obtained by sampling every second the rectangular components of the voltages and currents provided by time simulation. The system states were reconstructed with the same periodicity.

The load powers $P_{i}^{0}$ and $Q_{i}^{0}$ involved in the objective (4a) are the initial values of the corresponding load powers. The same values are used throughout all successive state reconstructions, with the aim of checking the quality of state reconstruction while the exact state is deviating from the reference state. The change in topology (line outage) in Scenario B is assumed to be known by the state reconstruction procedure.

Each optimization problem (4) was solved in the GAMSIDE (General Algebraic Modeling System) environment [17], interfaced with MATLAB [18] through the MATGAMS interface documented in [19]. The primal-dual interior-point nonlinear solver (with filter line-search method) IPOPT was used [20].

\section{B. Results for Scenario A}

The results for this scenario are illustrated in terms of reconstructed load active powers at buses 1041 (Fig. 3) and 2031 (Fig. 4). Both figures show the exact values (available at each integration time step) and the ones reconstructed from each of the three PMU configurations (available once per second). All values are in per unit on a 100-MVA base. Bus 1041 is where the step increase in load takes place, at $t=100 \mathrm{~s}$. Bus 2031 is located outside the Central region, and hence state reconstruction performance is expected to deteriorate for Configurations 2 and 3.

Figure 3 shows that state reconstruction tracks very accurately the system changes up to the instant of the step increase

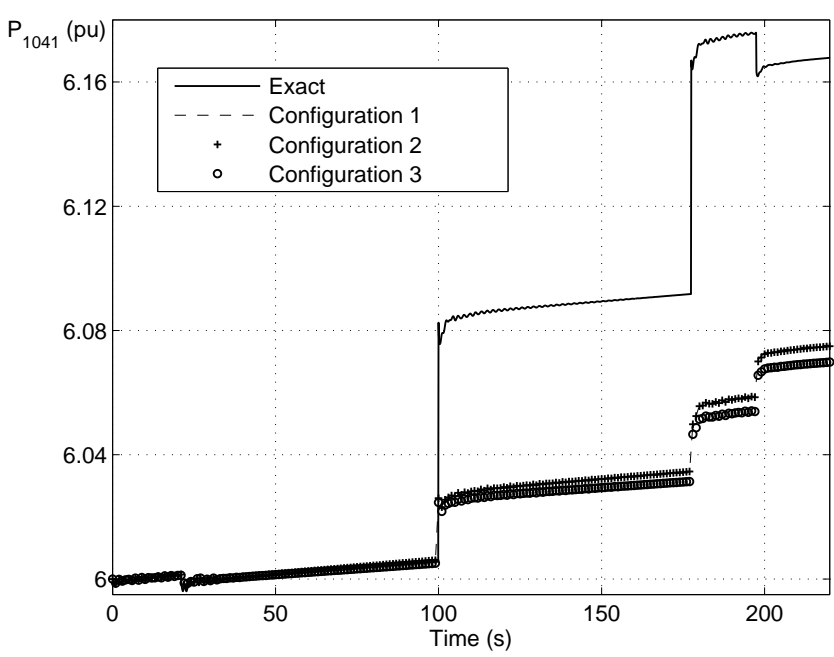

Fig. 3. Scenario A: exact and reconstructed load active power at bus 1041

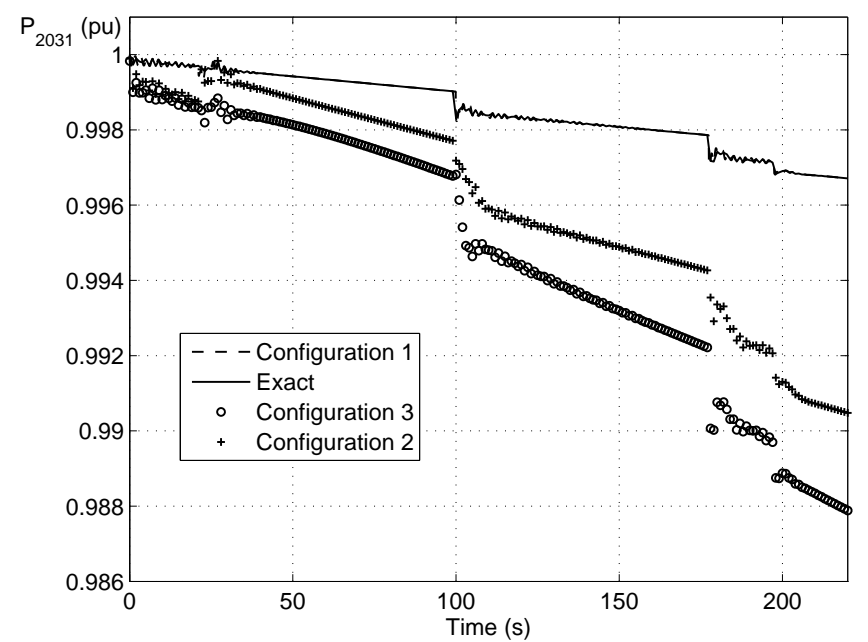

Fig. 4. Scenario A: exact and reconstructed load active power at bus 2031

in load demand. From there on, state reconstruction is still able to track changes but with a bias. The latter increases when the load power further changes at $t=177 \mathrm{~s}$ under the effect of the tap change in the distribution transformer feeding this load, but decreases at $t=196 \mathrm{~s}$ when the tap of another transformer changes. The results obtained with Configurations 1 and 2 are indiscernible. The results obtained with Configuration 3 are very close, which suggests that local state reconstruction is possible with the help of PMUs monitoring the zone of interest. The final error is 9.1 MW with Configurations 1 and 2 and $10 \mathrm{MW}$ with Configuration 3. This represents only $1.7 \%$ of the load power. It is noteworthy that the largest discrepancy $\left|P_{i}-P_{i}^{0}\right|$ is observed for the load active power at bus 1041 , which gives an indication of where changes took place.

Figure 4 shows that the (comparatively smaller) changes in load power at bus 2031 are very accurately tracked with Configuration 1 (the curves are indiscernible) while the other two configurations yield a drift with respect to the exact 
value, more pronounced with Configuration 3. This was to be expected since in Configuration 2 (resp. 3), there is no PMU at the nearby generator 8 (resp. generators 8 and 12). Yet, the final errors remain small: $1.3 \mathrm{MW}$ with Configuration 3 , which amounts to $1.3 \%$ of the load power.

\section{Results for Scenario B}

This scenario is illustrated in terms of reconstructed values of voltage magnitude at bus 1041 (Fig. 5), load active power at bus 1041 (Fig. 6), voltage magnitude at bus 2031 (Fig. 7) and current magnitude in line 4032-4042 (Fig. 8). The results are shown for PMU Configurations 2 and 3.

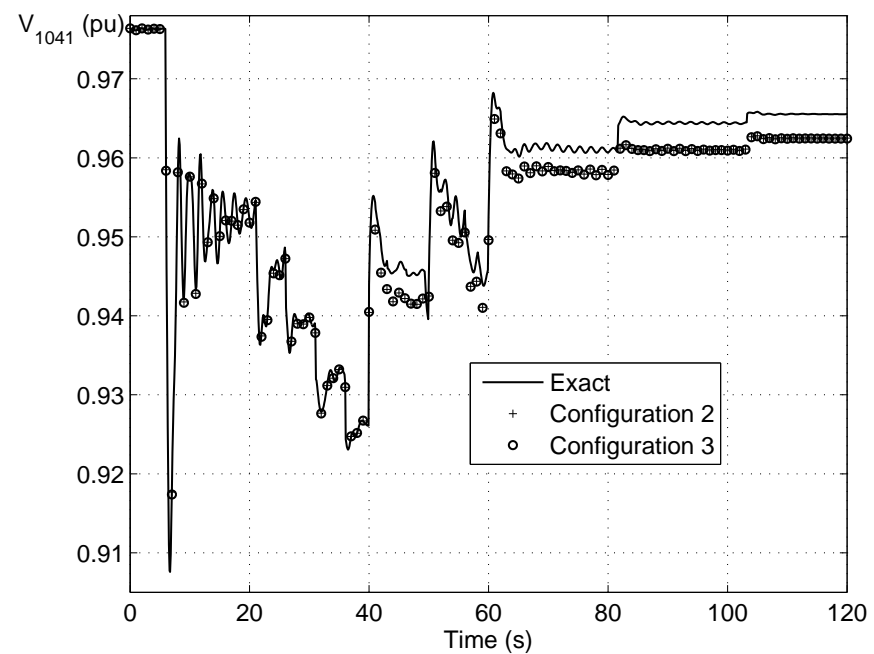

Fig. 5. Scenario B: exact and reconstructed voltage magnitude at bus 1041

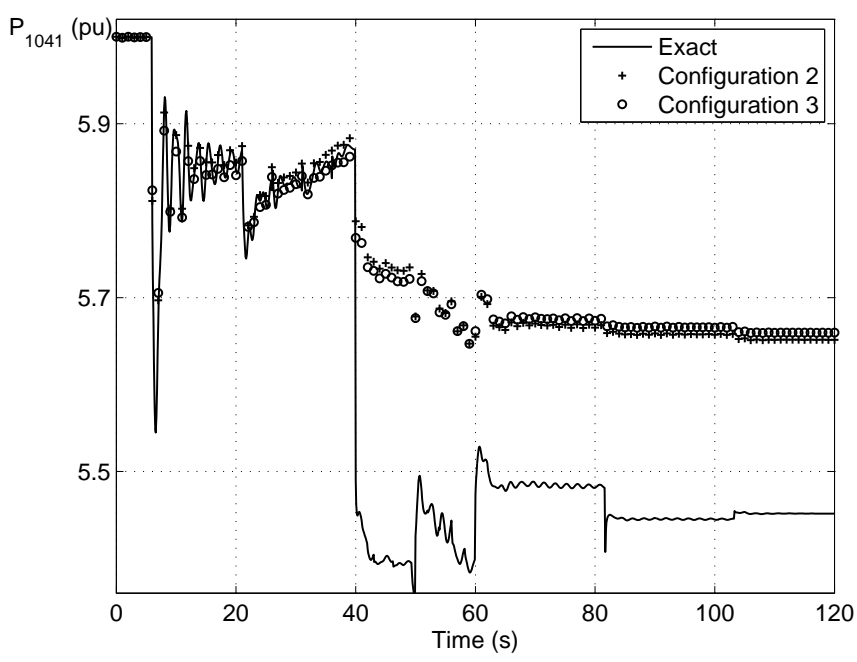

Fig. 6. Scenario B: exact and reconstructed load active power at bus 1041

Figures 5 and 6 show a remarkably accurate state reconstruction up to the time of the first load shedding. After that, the reconstructed state is a bit biased, but the overall accuracy remains quite good, with a final error of $0.004 \mathrm{pu}$ on the

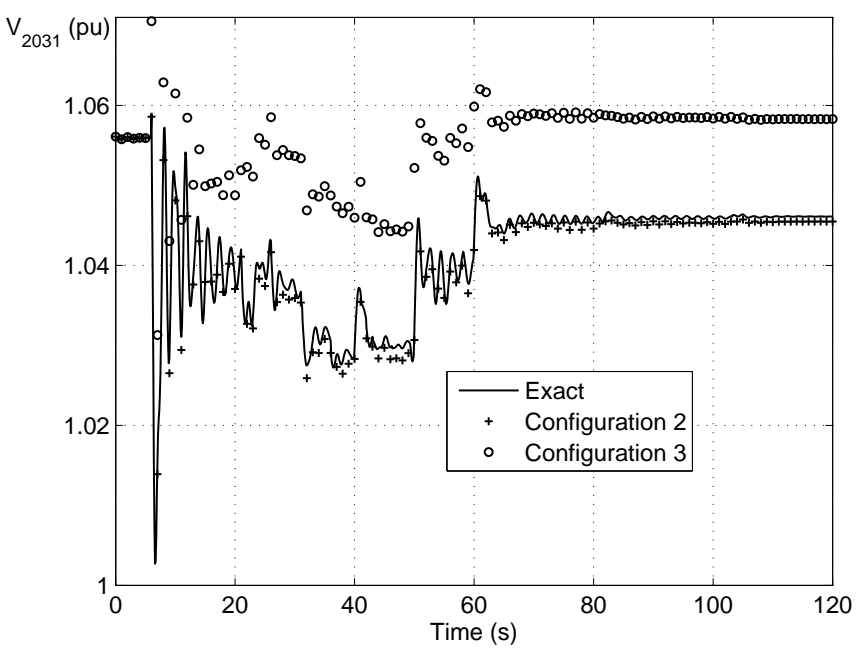

Fig. 7. Scenario B: exact and reconstructed voltage magnitude at bus 2031

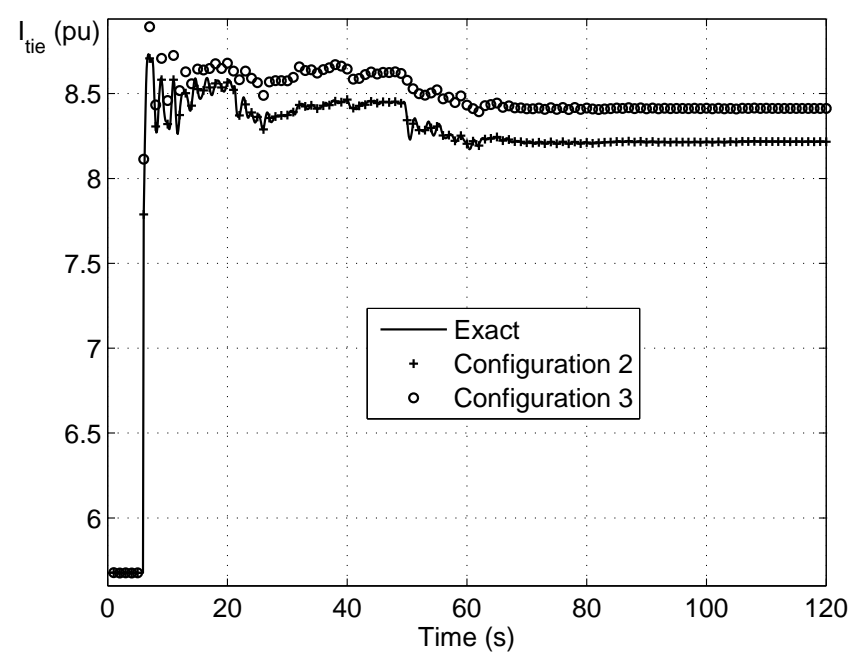

Fig. 8. Scenario B: exact and reconstructed current in line 4032-4042

voltage and $20 \mathrm{MW}$ on the active power. Again, these errors are caused by the fact that the reference power $P_{i}^{0}$ of this bus was kept equal to its pre-shedding value while it should be updated to reflect the load curtailment. It is interesting to see that the largest error on the reconstructed load power at bus 1041 takes place when this particular load is reduced, but changes relatively few (or even decreases) when the other two loads are curtailed. It is also noteworthy that Configurations 2 and 3 give basically the same results at this bus located in the region of interest.

As can be seen from Fig. 7, the voltage at bus 2031 is reconstructed with excellent accuracy when using Configuration 2. As expected, the error is more pronounced when using Configuration 3 (the final error is around $0.013 \mathrm{pu}$ ), since bus 2031 is now outside the region of interest monitored by PMUs.

Table IV shows the average error affecting the voltage magnitude, the voltage phase angle, and the active and reactive powers at a sample of buses, when using the scarce PMU data 
of Configuration 3. The results are the average errors over the 114 successive states reconstructed after the disturbance. As regards generators, the biggest errors take place in the North and Equiv areas, i.e. outside the region of interest, and are a consequence of not monitoring generators whose production is changing due to primary frequency control. As regards loads, the biggest errors correspond to buses 1043 and 1045, where the reference powers are not updated while load is curtailed.

TABLE IV

AVERAGE ERRORS ON RECONSTRUCTED VALUES (CONFIGURATION 3)

\begin{tabular}{|c|c|c|c|c|}
\hline & & \multicolumn{4}{|c|}{ average error on } \\
bus & $V(\mathrm{pu})$ & $\theta(\mathrm{rad})$ & $P(\mathrm{MW})$ & $Q$ (Mvar) \\
\hline g1 & 0.0567 & 0.0183 & $\mathbf{1 0 . 4}$ & $\mathbf{5 . 5}$ \\
g3 & 0.0565 & $\mathbf{0 . 0 2 4 3}$ & $\mathbf{8 . 8}$ & 2.7 \\
g4 & 0.0449 & $\mathbf{0 . 0 2 5 1}$ & 5.8 & $\mathbf{5 . 6}$ \\
g5 & 0.0341 & 0.0080 & 0.5 & $\mathbf{5 . 4}$ \\
g19 & $\mathbf{0 . 0 6 4 2}$ & 0.0085 & 0.2 & 0.4 \\
g20 & $\mathbf{0 . 0 6 9 3}$ & 0.0009 & $\mathbf{3 7 . 1}$ & 2.1 \\
1011 & 0.0548 & 0.0089 & 3.5 & 0.1 \\
1012 & 0.0564 & 0.0129 & 2.2 & 0.1 \\
2032 & 0.0131 & 0.0023 & 4.9 & 2.2 \\
1042 & 0.0001 & 0.0001 & 1.4 & 0.6 \\
1043 & 0.0004 & 0.0001 & 4.5 & 2.7 \\
1045 & 0.0016 & 0.0003 & $\mathbf{7 . 8}$ & $\mathbf{4 . 7}$ \\
\hline
\end{tabular}

One possible usage of state reconstruction is the anticipation of near-future system evolution and possible cascading effects of some disturbances. In this respect, it may of interest to quickly reconstruct the values of currents in important transmission lines. This is illustrated in Fig. 8, comparing the exact and reconstructed values of the current (magnitude) in line 4032-4042 located in the same corridor as the outaged line. Using Configuration 2, the value is reconstructed with remarkable accuracy. This can be explained by the presence of PMUs at the buses of generators g11, g12 and g14 located nearby on both sides of the line. With Configuration 3 , there is a degradation of accuracy but it remains quite moderate: in the order of $0.19 \mathrm{pu}$, to be compared to the $2.5 \mathrm{pu}$ increase due to the line outage. This result is even better that the only nearby PMU in Configuration 3 is on generator g14, i.e. only on one side of the line.

\section{CONCLUSION}

An approach to reconstruct system states from scarce synchronized phasor measurements has been proposed. The ideas leading to this approach can be summarized as follows:

- the PMU configuration provides scarce measurements, which do not make the system observable. As a consequence, there is an infinite number of states satisfying the available set of synchronized measurements;

- we solve this indeterminacy by computing the state which yields the bus power injections closest (in the Euclidiannorm sense) to reference values; the latter are the result of the last execution of a classical state estimator;

- this is equivalent to treating the reference power values as pseudo-measurements, in the least-square sense;

- this leads to solving an optimization problem with sparse linear equality constraints. The latter also include the zero injection information.
As regards PMU placement, it appears more appropriate to monitor generators. Indeed, it is preferable to monitor generator powers rather than load powers, in so far as the former vary more than the latter. Furthermore, when a generator is not monitored by a PMU, its complex power is free to vary in the optimization, and this may lead to an incorrect power exchange between this generator and some loads.

The main purpose of state reconstruction is to track the changing system state and fill the gap between successive classical state estimations by exploiting the coherent, timesynchronized phasor measurements. This could help monitoring the system after a large disturbance, in particular anticipating the near-future system evolution and possible cascading effects. State reconstruction could also replace a failing classical state estimation (owing to problems with bad data, divergence, etc.) or improve the accuracy of the latter when measurements suffer from bad data or time skew.

The simulation results obtained using snapshots provided by detailed time simulation have shown that:

- state reconstruction can be accurate with the help of PMUs monitoring generators;

- it is possible to accurately reconstruct the state of only a sub-network, using PMUs on the nearby generators. Outside the region of interest, the reconstructed state is less accurate, since it can only rely on the reference values of bus powers;

- the reconstructed state suffers from some bias when loads undergo significant changes, not reflected in the reference values.

The preliminary results are very encouraging. Of course, several issues and improvements deserve further investigation. Let us quote, non exhaustively:

- further test the capability of local state reconstruction. To this purpose, PMUs located on the tie-lines linking the region of interest to the rest of the system could supplement those on the nearby generators;

- investigate the possibility of updating the reference bus powers as time passes. The reconstructed state at one point in time could serve as reference for a few subsequent state reconstructions. This could help dealing with unexpected changes in load powers;

- test accuracy of state reconstruction for various levels of noise on PMU measurements;

- evaluate the benefits of state reconstruction to improve classical state estimation;

- investigate the best possible use of the PMU data gathered in between two state reconstructions;

- test the quality of the reconstructed state in demanding applications, such as voltage instability detection [22]

- devise an efficient mathematical programming algorithm to solve the optimization problem (4)

- envisage the addition of inequality constraints to (4), while preserving computational efficiency. 


\section{REFERENCES}

[1] A. G. Phadke, and J. S. Thorp, Synchronized Phasor Measurements and their Applicationa, Springer, 2008.

[2] D. Novosel, V. Madani, B. Bhargava, K. Vu, and J. Cole, "Dawn of the Grid Synchronization: Benefits, Practical Applications, and Deployment Strategies for Wide Area Monitoring, Protection, and Control," IEEE Power and Energy Magazine, vol. 6, no. 1, pp. 49-60, Jan. 2008.

[3] R. F. Nuqui, "State Estimation and Voltage Security Monitoring Using Synchronized Phasor Measurements," PhD Thesis, Virginia Polytechnic Institute and State University, Baslcksburg, Virginia, USA, Jul. 2001

[4] R. F. Nuqui and A. G. Phadke, "Phasor Measurement Unit Placement for Complete and Incomplete Observability," IEEE Trans. Power Del., Vol. 20, No. 4, pp. 2381-2388, Oct. 2005.

[5] T. L. Baldwin, L. Mili, M. B. Boisen, and R. Adapa, "Power System Observability With Minimal Phasor Measurement Placement," IEEE Trans. Power Syst., Vol. 8, No. 2, pp. 707-715, May 1993.

[6] L. Vanfretti, J. H. Chow, S. Sarawgi, B. Fardanesh, "A Phasor-Data-Based State Estimator Incorporating Phase Bias Correction," IEEE Trans. Power Syst., vol. 26, no. 1, pp. 111-119, Feb. 2011

[7] N. Zhou, Z. Huang, J. Nieplocha, T. B. Nguyen, "Wide-Area Situational Awareness of Power Grids with Limited Phasor Measurements," Proc. Third International Conference on Critical Infrastructures (CRIS), Alexandria, VA, Sept. 2006.

[8] T. Overbye, P. Sauer, C. DeMarco, B. Lesieutre, M. Venkatasubramanian, Using PMU Data to Increase Situational Awareness, Power System Engineering Research Center (PSERC) Publication 10-16, Sept. 2010.

[9] T.J. Overbye, J.D. Weber, "The Smart Grid and PMUs: Operational Challenges and Opportunities," Proc. IEEE PES 2010 General Meeting, Minneapolis, MN, July 2010.

[10] H. Liao, "Power System Harmonic State Estimation and Observability Analysis via Sparsity Maximization," IEEE Trans. Power Syst., vol. 22, no. 1, pp. 15-23, Feb. 2007.
[11] M. J. Smith, K. Wedeward, "Event Detection and Location in Electric Power System Using Constrained Oprimization,” Proc. IEEE PES 2009 General Meeting, Calgary, Canada, Jul. 2009.

[12] I. Roytelman, S. M. Shahidehpour, "State Estimation for Electric Power Distribution Systems in Quasi Real-Time Conditions," IEEE Trans. Power Del., vol. 8, no. 4, pp. 2009-2015, Oct. 1993.

[13] M. Dancre, P. Tournebise, P. Panciatici, F. Zaoui, "Optimal Power Flow applied to state estimation enhancement," Proc. 14th Power System Computation Conference (PSCC), Sevilla, Spain, Paper 37-2, Jun. 2002.

[14] P. C. Hansen, Rank-Defficient and Discrete Ill-Possed Problems: Numerical Aspects of Linear Inversion, Society of Industrial and Applied Mathematics (SIAM), 1998.

[15] T. L. Baldwin, L. Mili, M. B. Boisen, R. Adapa, "Power System Observability With Minimal Phasor Measurement Placement," IEEE Trans. Power Syst., vol. 8, no. 2, pp. 707-715, May 1993

[16] C. D. Vournas, E. G. Potamianakis, C. Moors, and T. Van Cutsem, "An educational simulation tool for power system control and stability," IEEE Trans. on Power Syst., vol. 19, No 1, pp. 48-55, Feb. 2004.

[17] [Online], Available: http://www.gams.com

[18] [Online], Available: http://www.mathworks.com

[19] M. C. Ferris, "MATLAB and GAMS: Interfacing optimization and vizualization software," [Online] Available: http://www.cs.wisc.edu/mathprog/matlab.html

[20] A. Wachter, L. T. Biegler, "On the Implementation of an Interior-Point Filter Line-Search Algorithm for Large-Scale Nonlinear Programming," Mathematical Programming, vol. 106, no. 1, pp. 25-57, 2006.

[21] M. Stubbe (Convener), Long-Term Dynamics - Phase II, Report of CIGRE Task Force 38.02.08, Jan. 1995.

[22] M. Glavic, T. Van Cutsem, "Wide-Area Detection of Voltage Instability From Synchronized Phasor Measurements. Part I: Principle. Part II: Simulation Results," IEEE Trans. Power Syst., Vol. 24, No. 3, pp. 14081425, Aug. 2009 